\title{
¿Noviembre azul? En ningún caso.
}

\author{
Blue november? In no case. \\ Novembro azul? Em nenhum caso.
}

Juan Gérvas (a)

El texto sobre "Um novembro não tão azul" ${ }^{1}$ es excelente, pero no es contundente. El programa de cribado de cáncer de próstata carece de fundamento científico y nunca se debería ofrecer como tal a toda la población de varones de 55 a 69 años (en otros grupos de edad no hay ni siquiera ensayos clínicos).

Para evaluar un programa de cribado (screening) de cáncer de próstata se necesitan cuatro datos:

1) la mortalidad por cáncer de próstata (es de esperar una disminución de la misma),

2) la calidad de vida de los pacientes sometidos al programa de cribado de cáncer de próstata (es de esperar una mejora de la calidad de vida),

3) la mortalidad general por cáncer (es de esperar una disminución de la misma), y

4) la mortalidad global, por todas las causas (es de esperar una disminución de la misma $)^{2-4}$.

El dato clave es el cuarto, el de la disminución de la mortalidad global, pues pudiera ser que el programa del cribado de cáncer de próstata disminuyera la mortalidad por dicho cáncer, que disminuyera la mortalidad general por cáncer y que incrementara la calidad de vida de los sometidos al programa pero que tuviera efectos adversos varios que llevasen al incremento de la mortalidad global. Si no disminuyese la mortalidad global el programa del cribado del cáncer de próstata sería un fracaso.

Pues bien, ningún programa de cribado de cáncer de próstata ha disminuido nunca la mortalidad global (ni ha aumentado la calidad de vida) ${ }^{5,6}$. De hecho, ningún programa de cribado de cáncer ha disminuido la mortalidad global ${ }^{7}$. Es decir, todos los cribados de cáncer han fracasado y deberían ser abandonados ${ }^{8}$, como se ha abandonado el cribado del neuroblastoma9.

En el mejor de los casos, en los ensayos clínicos en que se ofrece atención y seguimiento óptimos, un varón de 55 años integrado en un programa de cribado de cáncer de próstata con detección del PSA durante diez años verá disminuir su riesgo de muerte por cáncer de próstata del $0,6 \%$ al $0,5 \%(0,1 \%$ en riesgo

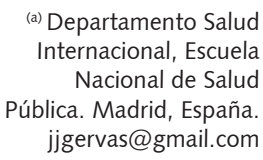


absoluto, $20 \%$ en riesgo relativo). Dicho varón tendrá un $10 \%$ de probabilidad de ser diagnosticado de cáncer de próstata gracias al programa de cribado, pero un $20-50 \%$ de esos cánceres hubieran sido silentes (in situ, sin dar nunca metástasis). Como consecuencia del tratamiento, dicho varón tendrá un $75 \%$ de posiblidades de padecer incontinencia urinaria, impotencia sexual o ambas ${ }^{10}$, lo que implica una grave disminución de la calidad de vida.

Se desconoce el impacto del tacto de próstata, que no se recomienda en ningún caso como parte de un programa de cribado de cáncer de próstata. Se desconoce el impacto del programa de cribado de cáncer de próstata en las condiciones "reales" de la práctica diaria.

Entre los grupos de riesgo se incluyen los varones negros y aquellos con familiares con cáncer de próstata. Puesto que la determinación del PSA lleva a mayor probabilidad de diagnóstico de cáncer de próstata, la recomendación de considerar grupo de riesgo a los varones que tengan familiares con cáncer de próstata sólo contribuye a crear una bola de nieve que crece sin cesar de varones diagnosticados de cáncer de próstata (sin impacto en la mortalidad global).

Por todo ello, es absurdo el "noviembre azul" y si interesa la salud de los varones habría que preocuparse todo el año por sus riesgos laborales, por los suicidios, por la violencia, por el tabaquismo y otras drogo-dependencias y en general por las condiciones socio-laborales que hacen que su expectativa de vida sea mucho menor que la de la mujer.

\section{Referencias}

1. Modesto AAD, Lima RLB, D'Angelis AC, Augusto DK. Um novembro não tão azul: debatendo rastreamento de câncer de próstata e saúde do homem. Interface (Botucatu). In press. doi: http://dx.doi.org/10.1590/1807-57622016.0288

2. Gigerenzer G. Full disclosure about cancer screening. BMJ. 2016; 352:6967.

3. Prasad V, Lenzer J, Newman DH. Why cancer screening has never been shown to "save lives"—and what we can do about it. BMJ. 2016; 352:6080.

4. Gérvas J. Ovarian cancer screening: could you recommend it? Evid Based Med. 2016; 21(4):134-5.

5. Dahm $P$, Neuberger $M$, Ilic D. Screening for prostate cancer: shaping the debate on benefits and harms. Cochrane Database Syst Rev. 2013; 9:ED000067.

6. Prasad V. The new recommendations for prostate cancer screenings are a bad deal [Internet]. 2017 [citado12 Abr 2017]. Disponível em: https://www.statnews. com/2017/04/11/psa-screening-prostate-cancer/.

7. Saquib N, Saquib J, loannidis JPA. Does screening for disease save lives in asymptomatic adults? Systematic review of meta-analyses and randomized trials. Int J Epidemiol. 2015; 44(1):264-77.

8. Gérvas J, Fernández MP. Cribados: una propuesta de racionalización. Gac Sanit. 2013; 27(4):372-3.

9. Tsubono Y, Hisamichi S. A halt to neuroblastoma screening in Japan. N Engl J Med 2004; 350(19):2010-1.

10. Bibbins-Domingo K, Grossman DC, Curry SJ. The US preventive services task force 2017 draft recommendation statement on screening for prostate cáncer: an invitation to review and comment. JAMA. 2017; 317(19):1949-50. 